\title{
Does coronary surgery prolong life in comparison with medical management?
}

\author{
ROBERT A. Rosati \\ M.D. \\ KERRY L. LEE \\ Ph.D. \\ Brant S. MitTleR \\ M.D. \\ J. FREDERICK MCNEER \\ M.D. \\ VICTOR S. BEHAR \\ M.D. \\ JAMES R. MARGOLIS \\ M.D.

\begin{abstract}
Division of Cardiology, Department of Medicine and the Department of Community Health Sciences, Duke University Medical Center, Durham, North Carolina
\end{abstract}

\begin{abstract}
Summary
This analysis attempts to utilize natural history controls to answer the question as to whether coronary surgery prolongs life in comparison to medical management. Selected natural history studies are compared and contrasted in an effort to obtain an average survival curve for patients with coronary artery disease comparable to those presently being operated. The Duke University Medical Center series of concurrent operated and non-operated patients is reviewed to demonstrate the difference of prognosis of patients with coronary artery disease and the complexities involved in answering the question. It is concluded that, on average, coronary surgery does not prolong life in comparison to medical management over the span of 2-5 years. There may be certain higher risk patients whose lives may be prolonged by aortocoronary bypass surgery but more patients and more prolonged followup are needed fully to answer the question.
\end{abstract}

THE resolution of the question of whether coronary artery bypass surgery can prolong life when compared to medical management involves the demonstration that survival in a group of operated patients is significantly higher than the survival in an adequate control group. The control, or non-operated, group must be sufficiently similar to the surgical group with regard to prognostically important descriptors that one would expect the same survival in both groups if neither was operated on.

There are two ways of obtaining such comparable groups. One is to allocate patients randomly to the two treatment groups in a prospective comparison,

This work was supported by Research Grants HS-01613, HL-17670, a contract NO1-HV-71440, a training grant LM-00149 and the Robert Wood Johnson Foundation.

Correspondence: Robert A. Rosati, Post Office Box 3337 , Duke University Medical Center, Durham, North Carolina 27710, U.S.A. and the other is to study operated and non-operated populations which have been sufficiently well described that matched groups can be selected from them. It is now proposed to discuss several reported series and present the Duke University Medical Center (DUMC) series of concurrent operated and non-operated patients.

\section{Reported series}

The survival curves of four operated and four nonoperated series are shown in Fig. 1. The operated groups are the Cleveland Clinic series of 1000 patients (Sheldon et al., 1973), the New York University (NYU) series of 448 patients (Spencer et al., 1974), the Texas Heart Institute (THI) series of 1105 patients (Cooley et al., 1973), and the Duke series of 490 patients. The non-operated groups are the Cleveland Clinic series of 469 patients (Sheldon et al., 1973; Webster, Moberg and Rincon, 1974), the Johns Hopkins series of 103 patients (Friesinger, Page and Ross, 1970; Humphries et al., 1974), the Queen's Hospital series of 259 patients (Burggraf and Parker, 1975), and the Duke series of 611 patients. There is considerable variation in the survival curves, but the operated series are not dramatically different from the non-operated series.

In Fig. 2 the operated series are shown separately. The curves differ in shape, primarily because of the operative mortality; but the 4-year survival is remarkably similar. The similarity is especially striking when one considers the stated differences in the patients reported. The NYU and THI series contain patients who had bypass only while the Cleveland and Duke series include patients who had ventricular and/or valve surgery as well. The Cleveland Clinic report does not state how patients were selected for surgery, but does state that a comparable medical series was selected by eliminating patients 'with 


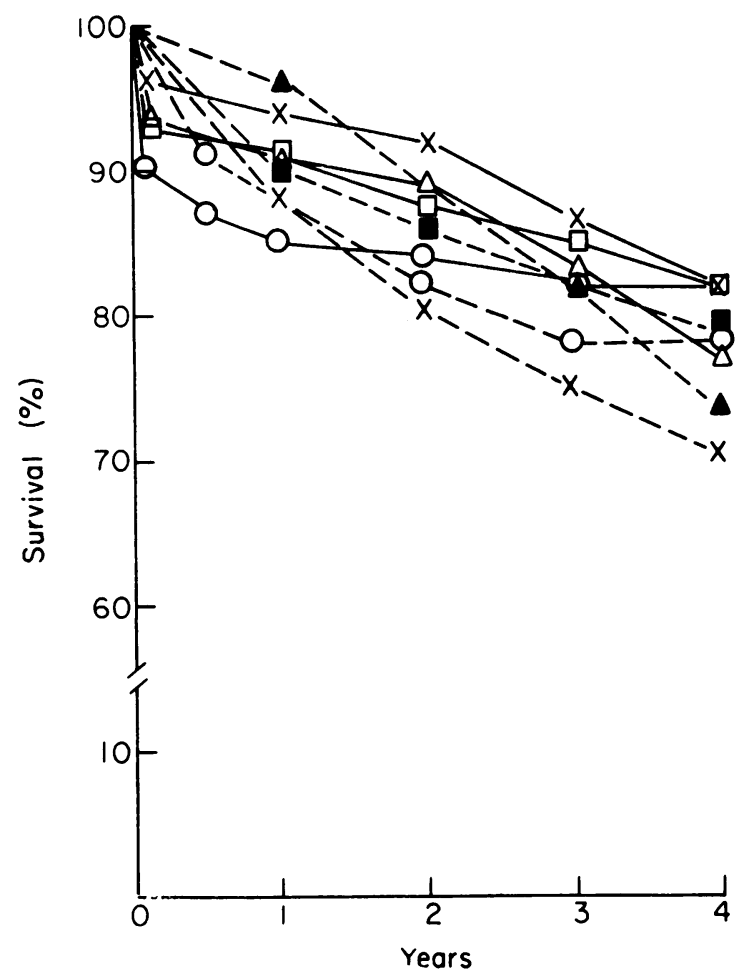

Fig. 1. Survival in coronary artery disease in selected operated and non-operated series. Operated: $x-\times$, Cleveland (1000 patients); $\triangle-\triangle$, NYU (448); $\square-\square$, THI (1105); $\bigcirc-O$, Duke(490). Non-operated: $\times---\times$, Cleveland (469); $\Delta---\Delta$, Hopkins (103); $\square---\square$, Queen's (259); O-- - O, Duke (611).

marked cardiomegaly, ventricular aneurysm, severe myocardial impairment as estimated by left ventriculography, a history of cardiac decompensation or associated valvular rheumatic disease'. The Duke series has been defined previously (McNeer et al., 1974 ) in terms of eighty-nine pre-surgical characteristics and in relation to the non-operated patients seen during the same time interval. Despite these obvious differences in patients operated, the survival rates in the surgical series are remarkably similar and the question of whether surgery prolongs life in any of these series depends on the medical series used as a control. As can be seen in Fig. 1, survival in all surgical series is better than the Cleveland Clinic nonoperated series, but none is significantly different from the Queen's Hospital or DUMC non-operated series.

Each of the non-operated series was reported in order to define the natural history of angiographically proved coronary artery disease so that the effect of bypass surgery on survival could be assessed. Unfortunately the non-operated series differ from one another both in the selection of patients and the

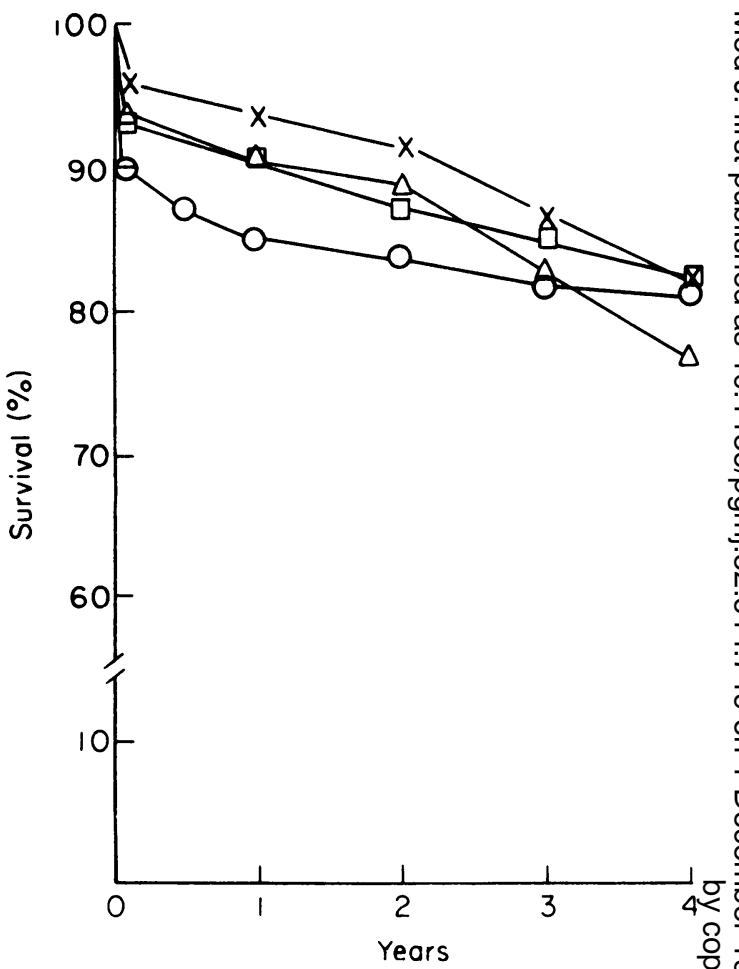

Fig. 2. Survival in coronary artery disease in selected series of patients who underwent aortocoronary bypass surgery. $x-\times$, Cleveland (1000 patients); $\triangle-\triangle$, ? NYU (448); $\square-\square$, THI (1105); ○-○, Duke (490).

results. The non-operated series are examined more closely in Tables 1-3. Table 1 indicates how the nonoperated series were accumulated. The patients in the Cleveland and Johns Hopkins series were catheterized before the advent of bypass surgery. The Queen's Hospital patients were catheterized more recently but still to a large extent before bypass surgery was available. The non-operated series at Duke was accumulated concurrently with a bypass series. This may be important when operated and non-operated groups are compared, as indications for investigation might well have changed after surgical treatment became possible.

Table 1 also shows that patients in the non-operated series were only a fraction of those catheterized

TABLE 1. Natural history studies

\begin{tabular}{lccc}
\hline & & \multicolumn{2}{c}{ No. of patients } \\
\cline { 3 - 4 } & catheterization & Catheterized & $\begin{array}{c}\text { Included } \\
\text { in study }\end{array}$ \\
\hline Cleveland & $1960-1965$ & 3527 & 469 \\
Hopkins & $1960-1967$ & 350 & 103 \\
Queen's & $1964-1970$ & 552 & 259 \\
Duke & $1969-1975$ & 1621 & 611 \\
\hline
\end{tabular}


TABLE 2. Natural history studies-exclusions

\begin{tabular}{lccccc}
\hline & $\begin{array}{c}\text { Associated } \\
\text { cardiac } \\
\text { disease }\end{array}$ & $\begin{array}{c}\text { Patients } \\
\text { treated } \\
\text { surgically }\end{array}$ & $\begin{array}{c}\text { Insignificant } \\
\text { CAD } \\
(\% \text { occlusion })\end{array}$ & $\begin{array}{c}\text { Anatomic } \\
\text { patterns }\end{array}$ & $\begin{array}{c}\text { Ventricular } \\
\text { impairment }\end{array}$ \\
\hline Cleveland & Yes & Yes & $<80$ & $\begin{array}{c}\text { Isolated } \\
\text { non-dominant } \\
\text { RCA disease }\end{array}$ & Yes \\
Hopkins & Yes & Yes & $<50$ & None & No \\
Queen's & Yes & Yes & $<50$ & $\begin{array}{c}\text { Left } \\
\text { main } \\
\text { disease }\end{array}$ & No \\
Duke & Yes & Yes & $<70$ & None & No \\
\hline
\end{tabular}

TABlE 3. Natural history studies-severity of disease and survival

\begin{tabular}{lccc}
\hline & $\begin{array}{c}\text { 1-vessel } \\
\text { disease } \\
(\%)\end{array}$ & $\begin{array}{c}3 \text {-vessel } \\
\text { disease } \\
(\%)\end{array}$ & $\begin{array}{c}\text { 4-year } \\
\text { survival } \\
(\%)\end{array}$ \\
\hline Cleveland & 38 & 24 & 71 \\
Hopkins & 31 & $45^{*}$ & 74 \\
Queen's & 39 & 25 & 79 \\
Duke & 22 & 54 & 78 \\
\hline
\end{tabular}

* Hopkins' score $\geqslant 10$.

and one must ask how they were selected. The answer to this question is attempted in Table 2. Patients with associated cardiac diseases such as rheumatic valvular heart disease and patients who subsequently underwent revascularization procedures were excluded from all series. The exclusion of patients who had revascularization procedures shortly after catheterization would introduce a bias if low risk patients were selected for surgery, so that non-operated patients would have had a worse prognosis than those selected for surgery. To assess this bias, one must know the indications for selecting patients for revascularization. In the Duke series, the comparability of the non-operated and operated patients was assessed (McNeer et al., 1974). In the other three series, the comparability of the non-operated patients and those who had revascularization procedures near the time of catheterization was not assessed. The other source of bias was the exclusion of patients who had bypass at a date remote from the time of their initial catheterization, as in the Cleveland, Hopkins and, probably, the Queen's series. This exclusion caused the reported survivals to be lower than they actually were because only patients who had survived many years were excluded, without any opportunity for patients who had died also to be excluded. The greater the number of patients subsequently bypassed, the lower the survival of the non-operated group would have been. This bias could have been minimized by the use of the life-table method (Cutler and Ederer, 1958), as in the Duke series. Patients who had bypass surgery more than 6 months after catheterization were counted as alive on each follow-up until the one before their surgery, and as withdrawn from the nonoperated group on the anniversary following surgery. With the life-table method, patients counted as withdrawn during any interval are considered to have a risk comparable to that of the patients whose outcome is known and the survival for the interval is adjusted accordingly.

Other exclusions might also have been expected to influence survival. All series excluded patients with insignificant or no coronary artery disease, but the definition of insignificant varied from less than $50 \%$ occlusion to less than $80 \%$ occlusion. The Cleveland series eliminated patients with isolated non-dominant right disease and patients with ventricular impairment as defined previously. The Queen's series analysed patients with left main coronary disease separately.

Finally, the inconsistencies between the nonoperated series are illustrated in Table 3 which shows the prevalence of single and 3-vessel disease in each series and relates these to 4-year survival. The Cleveland and Queen's patients were comparable in terms of the proportions with 1- and 3-vessel disease, yet differed significantly $(P<0.025)$ in 4-year survival, $71 \%$ and $79 \%$ respectively. The Hopkins series which had more severe vessel disease (if a score of $\geqslant 10$ is equated with 3-vessel disease) had an intermediate 4-year survival of approximately $74 \%$ (estimated from the survival curve published by Friesinger et al., 1970). The Duke series, which had the smallest proportion of single vessel disease and the most 3-vessel disease, had one of the higher 4year survivals, $78 \%$. This survival was not different from that reported in the Queen's series and was significantly different from the Cleveland series $(P<0.001)$. Thus, the survival rates reported in these series were not consistent considering the difference in severity of disease. These inconsistencies cannot be explained by the data presented in these otherwise 
TABLE 4. Interval survival data for operated and non-operated patients

\begin{tabular}{|c|c|c|c|c|c|c|}
\hline & \multicolumn{3}{|c|}{ Operated } & \multicolumn{3}{|c|}{ Non-operated } \\
\hline & Alive & Dead & Withdrawn* & Alive & Dead & Withdrawn* \\
\hline Surgical & 440 & 50 & 0 & & & \\
\hline 6 months & 419 & 14 & 7 & 554 & 53 & 4 \\
\hline 12 months & 374 & 8 & 37 & 480 & 18 & 56 \\
\hline 24 months & 295 & 5 & 74 & 291 & 26 & 163 \\
\hline 26 months & 199 & 5 & 91 & 147 & 12 & 132 \\
\hline 48 months & 76 & 1 & 122 & 53 & 0 & 94 \\
\hline
\end{tabular}

* Patients who had not reached anniversary date or were lost to follow-up.

well described series. There may be multifactorial differences which cannot be appreciated from the data presented, or there may be differences not described, such as why the patients were catheterized or how many survivors were subsequently eliminated.

In summary, the reported series illustrate the variability of patients with arteriographically proved coronary artery disease and emphasize how important it is that a non-operated series, when used as a control for an operated series, should be truly comparable.

\section{Duke University Medical Center Series}

In the remainder of the paper, the DUMC series of concurrent operated and non-operated patients will be discussed. The authors' approach has been to describe patients as well as possible and await the outcome before saying that their descriptions actually defined people similar enough to have a reproducible outcome; in this case, survival rate.

The data bank used in this analysis as well as the method of computing and comparing survival rates and of assessing comparability of operated and nonoperated groups has been described previously (McNeer et al., 1974; Rosati et al., 1975). The present analysis was performed on the 1101 consecutive patients with significant coronary artery disease who were catheterized at DUMC between August 1969 and September 1974. Significant coronary artery disease was defined as $70 \%$ or greater occlusion of at least one coronary artery. Follow-up data were obtained at 6 months, 12 months and annually thereafter, and were $>99 \%$ complete. Survival rates for operated and non-operated groups were calculated by the life-table method (Cutler and Ederer, 1958) and compared using the general linear model (Koch, Johnson and Tolley, 1972). Operated and nonoperated groups were examined to determine the extent of baseline inequalities in ninety-two zero-time characteristics.

There were 490 operated patients and 611 nonoperated patients. Table 4 shows the life-table survival data for these patients. Of the 611 non-operated patients, twenty-one were operated on more than 6 months after their initial catheterization. Figure 3 shows the survival curves for these concurrent groups. At 4 years, the survival rate was $82 \%$ in the operated patients and $78 \%$ in the non-operated patients. These survival rates were not significantly different. When the groups were compared, it was apparent that what difference there was could be attributed to baseline inequalities rather than the method of treatment. The same baseline inequalities reported in the first 781 of these patients (McNeer et al., 1974) were again observed. In addition, it was apparent that the non-operated group was older, and had an excess of abnormalities, electrocardiographic left ventricular hypertrophy, 3-vessel disease,

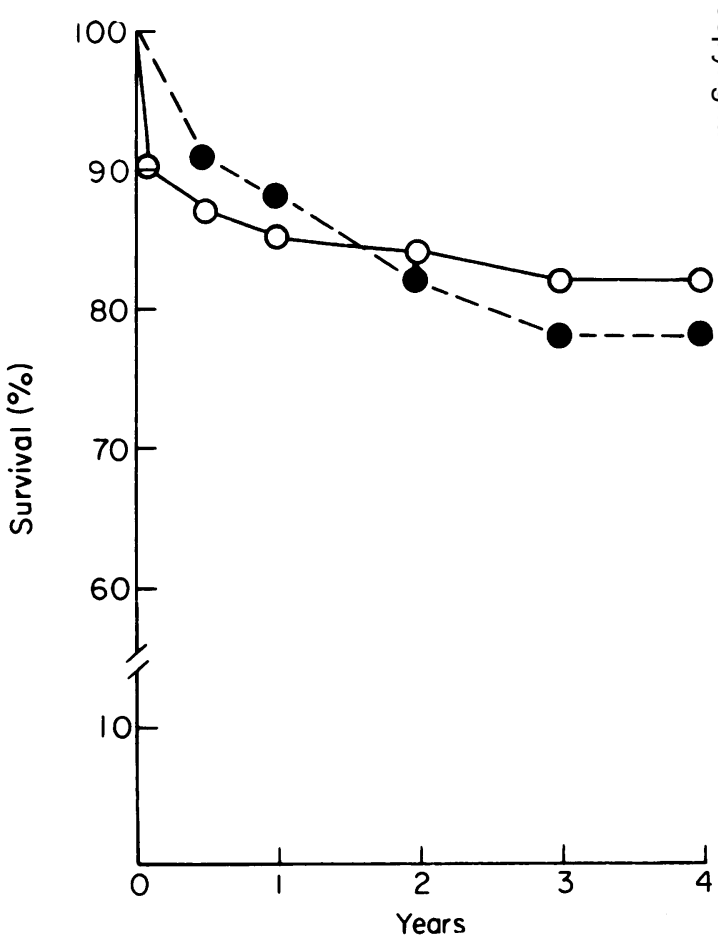

Fig. 3. Survival in coronary artery disease in the DUMC series of concurrent operated and non-operated patients. $\bigcirc-\bigcirc$, Operated (490 patients); $\bigcirc-$. , non-operated (611 patients). 


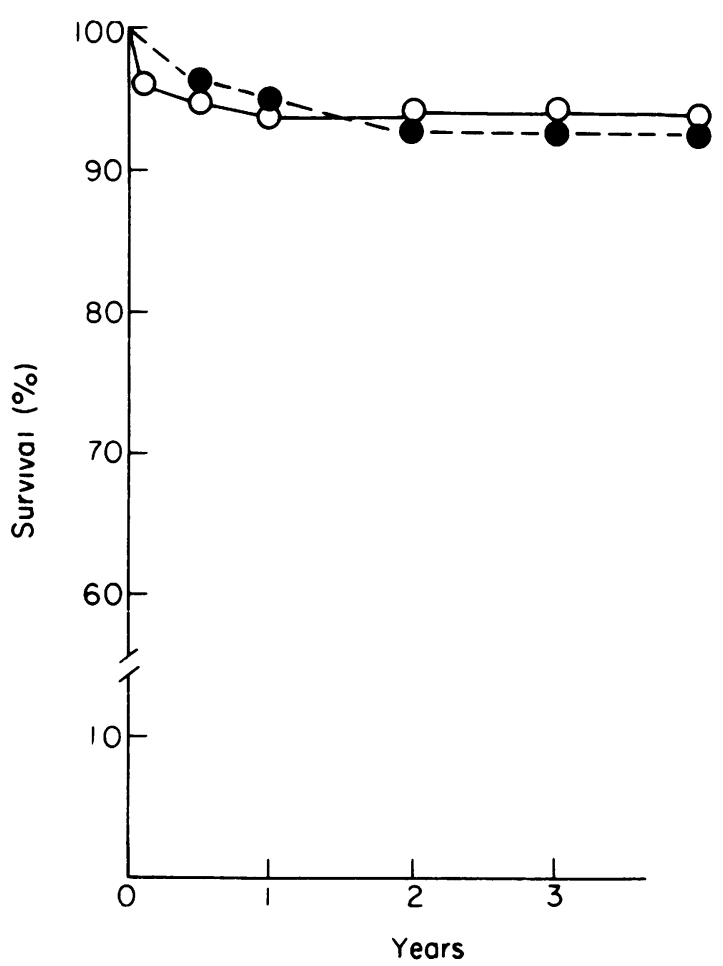

FIg. 4. Survival in coronary artery disease in patients who had no history of congestive heart failure, a normal left ventricular contraction pattern, no valvular heart disease and no left main coronary artery disease. $\bigcirc-O$, Operated (192 patients); --- 0 , non-operated (225 patients).

distal vessel disease, abnormal left ventricular contraction patterns and elevated left ventricular end diastolic pressures in comparison to the operated group. Selected prognostically important baseline inequalities are shown in Table 5.

TABLE 5. Significant baseline inequalities

\begin{tabular}{lcc}
\hline & \multicolumn{2}{c}{ Prevalence (\%) } \\
& Operated & Non-operated \\
\hline Age $\geqslant 60$ years & 11 & 17 \\
Cardiomegaly, chest X-ray & 13 & 23 \\
LVEDP $\geqslant 18$ mmHg & 10 & 19 \\
LV contraction, abnormal & 49 & 56 \\
Mitral insufficiency & 9 & 17 \\
3-Vessel disease & 49 & 54 \\
Distal vessel disease & 52 & 64 \\
\hline
\end{tabular}

Thus, an increase in the number of patients observed is unlikely to make a significant difference in the result. It is probable that for the average patient with coronary artery disease, bypass surgery does not significantly prolong life over a 4-year period. But one never has to decide how to treat the average patient.
What one needs to know is what will happen to a specific individual patient if he is treated surgically and what will happen if he is treated medically. When these outcomes for individual patients are known, whether surgery will prolong life for the individual patient will also be known.

The closest we can come to knowing what will happen to the new patient is to know what has happened to patients like him. The difficulty comes in being sure that the past patients are like the new patient and that we can, therefore, expect the same outcome. If a new patient is low risk, he is recognized by a series of normal characteristics. Since prognostically, there is a narrow spectrum of normality, patients without abnormal characteristics are likely to be homogeneous. Take, for example, a sub-group of patients defined by a combination of reported criteria for low-risk surgical patients. Such a subgroup might be defined as having no history of congestive heart failure, normal left ventricular contraction as assessed by ventriculography, no valvular heart disease and no left main disease. Figure 4 illustrates the survival curves of these patients. The 4year survival rates in the operated and non-operated groups were $94 \%$ and $93 \%$ respectively. The surgical groups had more severe and frequent pain which was more often progressive and more often typical angina and had more often been treated with $\beta$-blocking agents than the non-operated group. Correction for these inequalities did not appreciably affect the survival rates. The addition of one or two pain characteristics would have made these groups very similar. While it is possible that surgery may prolong life in these low-risk patients, it is clear that they will have to be followed for many more than 4 years before this effect will become apparent.

Patients who are at high risk present a slightly different problem. They are characterized by abnormalities. Since there is a wide spectrum of abnormality as opposed to the narrow spectrum of normality, patients characterized by abnormalities are likely to be non-homogeneous unless these abnormalities are further defined. For example, consider the sub-group of patients which were reported previously (McNeer et al., 1974) as having a higher survival if treated surgically than if treated medically. This sub-group had a normal arteriovenous oxygen difference, an abnormal left ventricular contraction pattern and 3-vessel disease. The 4-year survival rates of the original sub-group of operated and nonoperated patients were $84 \%$ and $70 \%$ respectively (Fig. 5). Figure 6 shows the 4-year survival curves for this same sub-group from September 1974 with the 100 additional patients who have been seen since the previous report. The 4-year survival of the operated group is $79 \%$ and that of the non-operated group is $74 \%$. These rates are not significantly different. 


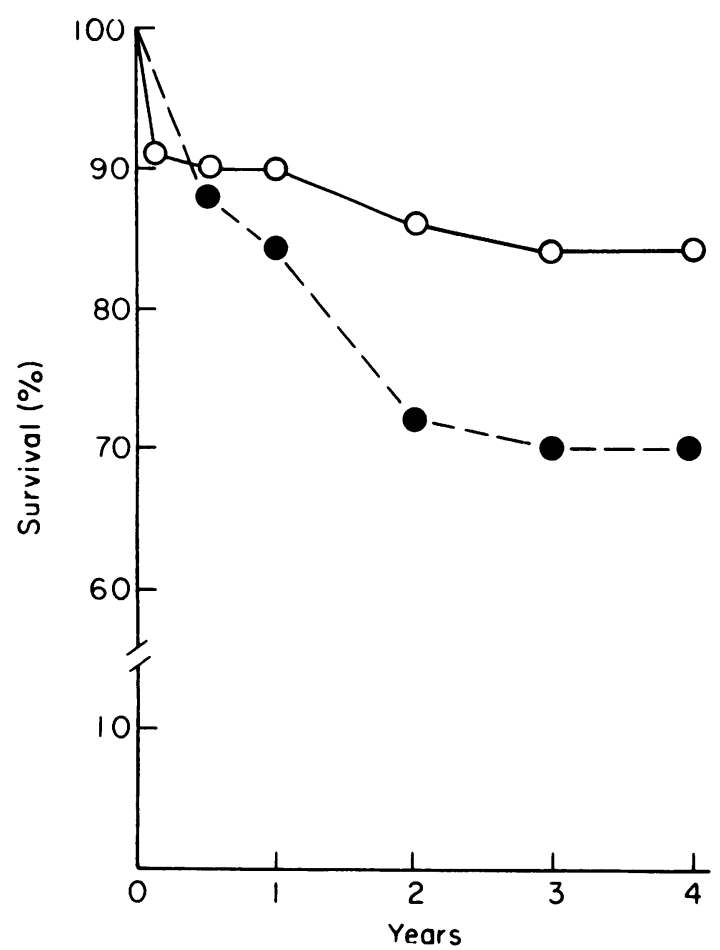

FIG. 5. Survival in coronary artery disease in patients catheterized before April 201973 (sub-group F, 4/73) who had an arteriovenous oxygen difference of $\leqslant 5.5$ vol. $\%$, an abnormal left ventricular contraction pattern and 3-vessel disease. $\bigcirc-O$, Operated (73 patients);

- - - non-operated (96 patients).

There are two possible reasons for this apparent change in results. First, the original groups may not have been as similar as at first thought. The nonoperated group shown in Fig. 6 continued to have greater proportions of diffusely abnormal ventricular contraction patterns and totally occluded left circumflex coronary arteries. Since the original report, the other differences apparent at that time (April 1973) had become insignificant, and characteristics which were not significantly different at that time had become significantly different. The non-operated group, from September 1974, was slightly older than the operated group and had significantly fewer good distal vessels than the surgical group. The difference in distal vessel disease was attributed to an excess of good right coronary and circumflex distal vessels in the operated group. A re-analysis of the original sub-group, as it was constituted in April 1973, showed that in patients with three good distal vessels, survival in the non-operated group was higher than in the operated group. This analysis was not performed originally because there was no statistically significant difference in distal vessel disease at that time.

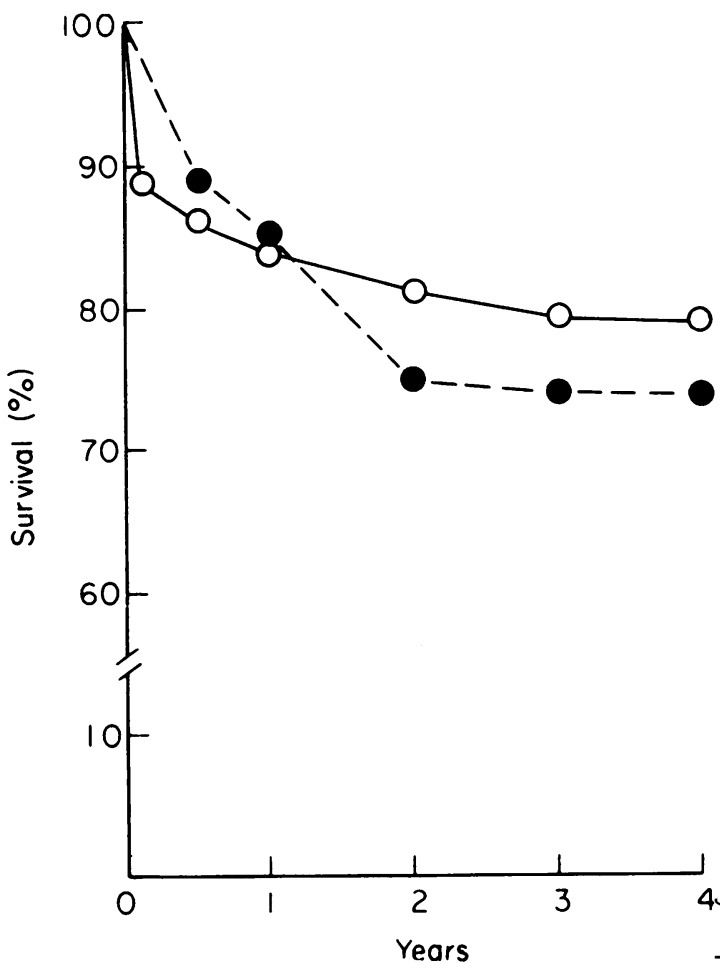

FIG. 6. Survival in coronary artery disease in patients catheterized before September 1974 (sub-group F, 9/74) who had an arteriovenous oxygen difference of $\leqslant 5.5$ vol. $\%$, an abnormal left ventricular contraction pattern and 3 -vessel disease. $\bigcirc-O$, Operated (105 patients); -. - . non-operated (164 patients).

A second possibility is that the 100 patients seen since April 1973 were different from those seen before April 1973. Comparison of patients in the original sub-group to those catheterized subsequently revealed a general tendency toward selection of lower risk patients for catheterization and several extremely ill operated patients with no non-operated counterparts in the more recent series. In either case, the patients in this sub-group were not sufficiently distinguished by these three characteristics to be homogeneous and come to common end points.

The approach here must be to distinguish patients by an increasing number of characteristics in order to obtain increasingly homogeneous groups. In this way, the new patient, and his doctor, can be told what has happened to others like him, when treated medically or surgically. Table 6 illustrates a prognostic report devised to convey this information. The first section contains the description of a new patient (A). He is similar to patients contained in the sub-group in which surgery had formerly appeared to prolong survival (3-vessel disease, abnormal ventricular contraction pattern and normal arterio- 
TABLE 6. A prognostigram

For index case: patient $\mathrm{A}$

Prognostrigram

With the following criteria:

3-Vessel coronary artery disease

No significant left main coronary disease

Total occlusion of the right coronary artery

Sub-total occlusion of the left anterior descending coronary artery

Good distal left anterior descending

Localized left ventricular contraction abnormality, not anterior

No ventricular aneurysm

No mitral insufficiency

Normal arteriovenous oxygen difference

A sub-group of twenty-three patients was found

Prognostic tabulation

\begin{tabular}{lccccccccc}
\hline \multicolumn{3}{c}{ Medicine } & \multicolumn{5}{c}{ Surgery } \\
\hline & Alive & Dead & *NRA & Survival \% & Alive & Dead & *NRA & Survival \% & $P$ \\
Surgical & & & & & 11 & 0 & 0 & $100 \cdot 0$ & \\
6-Month & 9 & 3 & 0 & 75.0 & 11 & 0 & 0 & $100 \cdot 0$ & 0.08 \\
1-Year & 9 & 0 & 0 & 75.0 & 11 & 0 & 0 & $100 \cdot 0$ & 0.08 \\
2-Year & 3 & 1 & 5 & 63.5 & 9 & 0 & 2 & 100.0 & 0.05 \\
3-Year & 1 & 0 & 2 & 63.5 & 4 & 0 & 5 & 100.0 & \\
4-Year & 1 & 0 & 0 & 63.5 & 1 & 0 & 3 & 100.0 & \\
\hline
\end{tabular}

*NRA-not yet reached anniversary

venous oxygen difference), but those parameters which are abnormal are more exactly defined. Not only has he 3-vessel disease but also no significant left main disease, a total occlusion of the right coronary artery, and a sub-total occlusion of the left anterior descending with a good distal vessel. His left ventricular contraction abnormality is localized, is not an aneurysm and is not in the distribution of the left anterior descending. He has no mitral insufficiency and his arteriovenous oxygen difference is normal. There were twenty-three such patients in the data bank of 1101 patients. The second section gives the prognosis of these twenty-three patients. Nine of the patients had been treated medically and four had died so that the 4-year survival rate was $64 \%$. Eleven patients had been treated surgically with no deaths. Although the survival in this small subgroup is significantly higher in the surgical group at 2 years, only more observations will tell us whether this difference persists and whether the groups are similar enough to conclude that surgery prolongs life.

\section{Discussion}

It has been demonstrated that adequate characterization of patients permits comparisons to be made which indicate that, on average, coronary artery surgery does not significantly prolong 4-year survival when compared to medical treatment. The phrase 'on average' is most important. One might obtain comparable survival rates if surgery had no effect on survival in any patient. Alternatively, one might obtain comparable survival rates if surgery prolonged life in certain patients, shortened it in some and had no effect on survival in others. The latter possibility is most likely to be the case in a chronic disease such as coronary artery disease.

Four years is a short time in the life of the patient with coronary artery disease. To find differences in 4-year survival, we must look at relatively high risk patients and these are more likely to be non-homogeneous when defined by relatively few characteristics. When a sub-group with abnormal left ventricular contraction pattern is defined, room is allowed for many degrees of abnormality. If the abnormalities are further described, the sub-groups become small and time must pass for the numbers to increase. On the other hand, when we look at relatively low-risk patients such as a sub-group with normal left ventricular contraction patterns, there is left much less room for non-homogeneity; but there are few deaths within 4 years and we have to wait longer before it can be demonstrated that survival is affected by surgery.

It is apparent that it is relatively easy and takes a relatively small number of patients to answer the question of whether a treatment, on average, affects survival in a chronic disease. To answer the question of whether the treatment will prolong the life of a given patient is much harder and requires accurate characterization of many patients and follow-up over long periods of time. 


\section{References}

Burggraf, G.W. \& Parker, J.O. (1975) Prognosis in coronary artery disease. Angiographic, hemodynamic, and clinical factors. Circulation, 51, 146.

Cooley, D.A., Dawson, J.T., Hallman, G.L., Sandiford, F.M., Wukasch, D.C., Garcia, E. \& Hall, R.J. (1973) Aortocoronary saphenous vein bypass. Results in 1,492 patients with particular reference to patients with complicating features. Annals of Thoracic Surgery, 16, 380.

CUTLER, S.J. \& EDerer, F. (1958) Maximum utilization of the life table method in analyzing survival. Journal of Chronic Disease, 8, 699.

Friesinger, G.C., PAGe, E.E. \& Ross, R.S. (1970) Prognostic significance of coronary arteriography. Transactions of the Association of American Physicians, 83, 78.

Humphries, J.O., Kuller, L., Ross, R.S., Friesinger, G.C. \& PAGE, E.E. (1974) Natural history of ischemic heart disease in relation to arteriographic findings. A twelve year study of 224 patients. Circulation, 49, 489.

Koch, G.G., Johnson, W.D. \& Tolley, H.D. (1972) A linear models approach to the analysis of survival and extent of disease in multidimensional contingency tables. Journal of the American Statistical Association, 67, 783.
McNeer, J.F., Starmer, C.F., Bartel, A.G., Behar, V.S., Kong, Y., Peter, R.H. \& Rosati, R.A. (1974) The nature of treatment selection in coronary artery disease. Experience with medical and surgical treatment of a chronic disease. Circulation, 49, 606.

Rosati, R.A., McNeer, J.F., Starmer, C.F., Mittler, B.S. MorRis, J.J. \& Wallace, A.G. (1975) A new information system for medical practice. Archives of Internal Medicine, 135, 1017.

Sheldon, W.C., Rincon, G., Effler, D.B., Proudfit, W.L \& SONES, F.M., Jr (1973) Vein graft surgery for coronary artery disease. Survival and angiographic results in 1,C00 patients. Circulation, 47 (Suppl. 3), 184.

Spencer, F.C., Isom, O.W., Glassman, E., Boyd, A.D. Engleman, R.M., Reed, G.E., Pasternack, B.S. \& Dembrow, J.M. (1974) The long-term influence of coronary bypass grafts on myocardial infarction and survival. Annals of Surgery, 180, 439

Webster, J.S., Moberg, C. \& Rincon, G. (1974) Natural history of severe proximal coronary artery disease as documented by coronary cineangiography. American Journal of Cardiology, 33, 195. 